\title{
A mini review of haloalkane dehalogenase: From molecular characterization to applications
}

\author{
Khomaini Hasan* \\ Faculty of Medicine, Universitas Jenderal Achmad Yani, Terusan Jenderal Sudirman PO.Box 148, Cimahi, 40285, Indonesia
}

Article history:

Received: 19 January 2018 / Received in revised form: 30 May 2018 / Accepted: 30 May 2018

\begin{abstract}
Haloalkane dehalogenases (HLDs) are hydrolytic enzymes that catalyze a removal of halogenated species in many toxic halogenated compounds. These enzymes belong to hydrolase family that mostly adopt a typically $\alpha / \beta$ hydrolase structure. They have many potential applications, such as industrial biocatalysis, pharmaceutics, biosensors, or detoxification of chemical weapons. In this review, structure, mechanism and applications of these enzymes will be discussed.
\end{abstract}

Keywords: Haloalkane dehalogenase; biocatalysis; $\alpha / \beta$-hydrolase; industrial enzyme; halogenated compounds

\section{Introduction}

Haloalkane dehalogenases (HLDs, EC 3.8.1.5) are enzymes that catalyse the hydrolysis of carbon-halogen bonds of halogenated compounds, to produce a alcohol as the main products. These enzymes are active toward halogenated alkanes, cycloalkanes, alkenes, ethers, alcohol, ketones or cyclic dienes [1-2]. The HLDs have great interest due to their potential applications on industrial biocatalysts, biosensor, or bioremediation [3]. The very first complete-characterized HLD was isolated from Xanthobacter autothropicus GJ10 in the mid-1980s [4]. Shortly afterwards, many HLDs from different sources are continuously identified and characterized, such as from symbiotic bacteria [5], pathogenic bacteria [6-7] or extremophiles [8].

\section{Structure and Mechanism}

The first HLD structure was reported by Dijsktra and coworker [9]. To date, several native structures of HLDs have been published and made available in Protein Data Bank. They include DhlA isolated from $X$. authotropicus GJ10 [9], DhaA from $R$. rhodochrous NCIMB13064 [5], LinB from $S$. paucimobilis UT26 [2], DmbA from M. tuberculosis Rv2579 [10], DbjA from B. japonicum USDA110 [11], DppA from P. pacifica SIR-1 [12], DmmA from M. producta [13] and DatA from Agrobacterium tumefaciens C58 [14].

All HLD structures share similar $\alpha / \beta$-hydrolase structure (Fig. 1), one of largest group of protein structure and highly conserved structure but exhibit an extraordinary diversity in sequence, catalytic mechanism and biochemical function. In

* Corresponding author.

Email: k.hasan@lecture.unjani.ac.id general, structure of HLDs consists of main domain with an eight-stranded parallel $\beta$-sheet structure and; connected by loops; $\alpha$-helices cap domain on top of main domain. The main domain and cap domain form a strong internal hydrophobic cavity which is deeply buried inside the core of the enzyme [2,5,9-10,12-16].

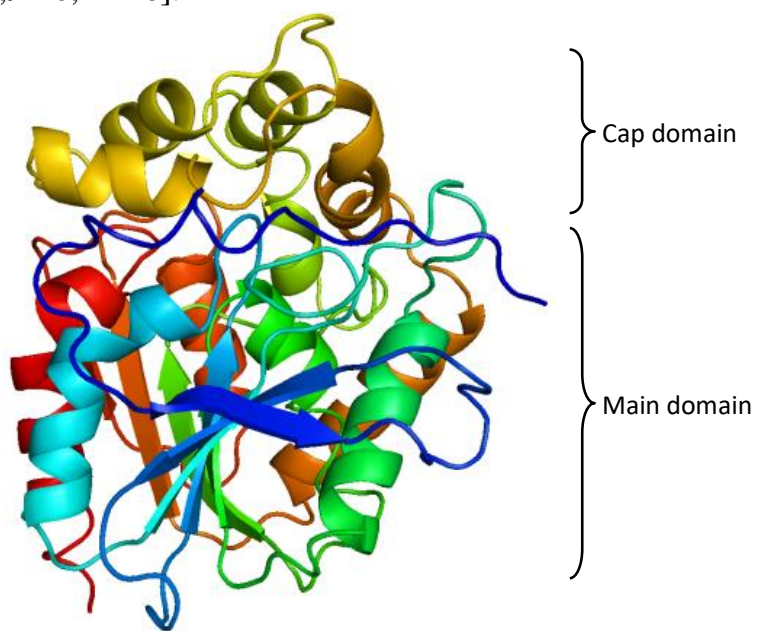

Fig. 1. Representative structure of haloalkane dehalogenase. PDB ID: 2HAD

The $\alpha / \beta$-hydrolase domain fold are the most frequently found in nature and are adopted by largest groups of proteins that display an enormous diversity in sequence, fold plasticity and catalytic functions and, moreover, in the evolution point of view, this fold diverge from a common ancestor [17-20]. This fold is a stable that can accommodate and shield an active site which is buried in the hydrophobic cavity of enzymes [18,20-21]. The $\alpha / \beta$-hydrolase domain fold preserves a typically elegant arrangement of active center at specific positions in the $\alpha / \beta$-hydrolase-fold motif bearing a conserved nucleophile-histidine-acid catalytic triad evolved to 
efficiently function scheme as, predominantly, hydrolase or esterases $[1,18,20]$.

Second domain, cap domain, is a $\alpha$-helices-rich domain that inserted and connected the C-terminally to strand $\beta 6$, to the main domain by variable length of flexible loops. These insertions contribute in the lining of the active-site cavity and a substrate-traffic tunnel. Due to their flexibility, essential concerted motions of this domain determine substrate interaction and product release; therefore, make this domain solely plays an important role in halide release and substrate specificity modulation $[5,11,15,22-25]$. The suggestion that a flexible motion of the cap domain involved in halide release and binding as well as determinant factor for substrate specificity had been supported by scientific evidences [25-26]. Moreover, the sequence and structure of the cap domain, for instance spatial arrangement between HLD-subfamily, greatly vary among different HLDs that influence the binding capability and kinetics of different substrates [22-24].

The active sites of HLDs are located in between the main domain and the cap domain which are protected from the solvent by fully hydrophobic residues, thus it is advantageous for the dehalogenation reaction $[3,15,27]$. The active site residues that are directly involved in the dehalogenation reaction constitute a catalytic pentad. Three residues, highly conserved residues for $\alpha / \beta$ hydrolases activity referred to as catalytic triad, consists of a nucleophilic aspartic acid residue, a basic histidine residue, an aspartic or glutamic acid moiety that serves as a general acid. The role of pair remaining residues serves as halide stabilizing residues [1,21] The composition of the catalytic pentad varies among different subfamily: Asp-His-Asp + Trp-Trp in HLD-I, Asp-His-Glu + Asn-Trp in HLD-II, and Asp-His-Asp + Asn-Trp in HLD-III $[22,28]$. Among subfamilies, they also have difference in the geometry and size of the active site cavity and how the leaving group is stabilized $[5,15]$.

In $\alpha / \beta$-hydrolase-family, a nucleophile positioned after strand $\beta 5$ on a very sharp turn, known as the nucleophile elbow, an acidic residue almost always positioned after strand $\beta 7$, and conserved histidine residue located after the last $\beta$ strand $[18,28]$. Two members of the catalytic triad, which are nucleophile and the histidine base, showed a very high level of rigidity in enzyme structure. This rigidity is favourable for the catalysis [24]. Meanwhile, two halide stabilizing residues which point towards the cavity are located in interface between the main domain and the cap domain [5-15].
A strictly conserved nucleophilic aspartic acid acts as nucleophile displacing a halide ion from the halogenated substrate [28]. Histidine, which is conserved residue also in serine proteases of the chymotrypsin and the subtilisin classes, roles in dealkylation of the covalent intermediate, which is formed by the nucleophilic attack, and to increase water molecule nucleophilicity that close to the carbonyl carbon of nucleophilic [29]. A catalytic acid represented by glutamic or aspartic acids play a role in keeping proper orientation of histidine and stabilises a positive charge that develops on histidine imidazole ring during the reaction. All catalytic triad operate as an electronic pump during the dehalogenation reaction $[28,30]$.

Furthermore, first halide stabilizing residue is located adjacent to the nucleophile, and second is localized in an $\mathrm{N}$ terminal helix $\alpha 4$ of cap domain, or on the loop between strand $\beta 3$ and the helix $\alpha 1$. They play significant role in the halide binding; via hydrogen bond; and maintain nucleophile in proper orientation, usually serves by highly conserved hydrophobic residue i.e. tryptophan [12-13,27,31]. Variability of first halide stabilizing residue was firstly observed in HLD, DatA, from A. tumefaciens C58. Sequence alignment revealed that halide-stabilizing residue adjacent to nucleophile is a tyrosine rather than the tryptophan observed in other members of the family [7,22]. The two halide stabilizing residues contribute significant effect on stabilization efficiency. Calculation of the halide stabilization efficiency of HLD revealed that trends in halide stabilization efficiency of different residues is in the order tryptophan > asparagines $>>$ tyrosine, and stabilization of halide stabilizing pair is in order Trp-Trp > Trp-Asn > Trp-Tyr [27].

Dijkstra and co-worker proposed the first detailed structure-based catalytic mechanism of HLDs [16]. Dehalogenation performs by two major steps reaction: first, by nucleophilic attack of an aspartic acid on the $\mathrm{sp}^{3}$ hybridised carbon atom that attached to the halogen as leaving group of the substrate in the reversible bimolecular nucleophilic substitution $\left(\mathrm{S}_{\mathrm{N}} 2\right)$-type reaction. It leads to cleavage of the halogen as halide and formation of a covalently bound alkyl-enzyme intermediate. During catalysis, a catalytic acid, represent by aspartic or glutamic acids, roles in polarizing histidine ring and stabilizing the positive charge as well as keeping proper orientation of the imidazole ring of the histidine during the hydrolytic reaction (Fig. 2) [28,30].

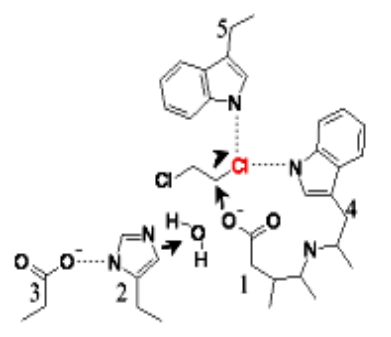

Nucleophilic attack

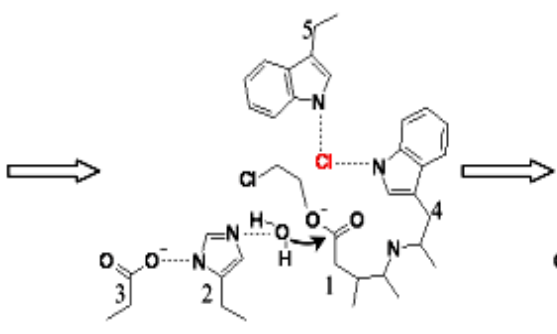

Alkyl enzyme intermediate formation

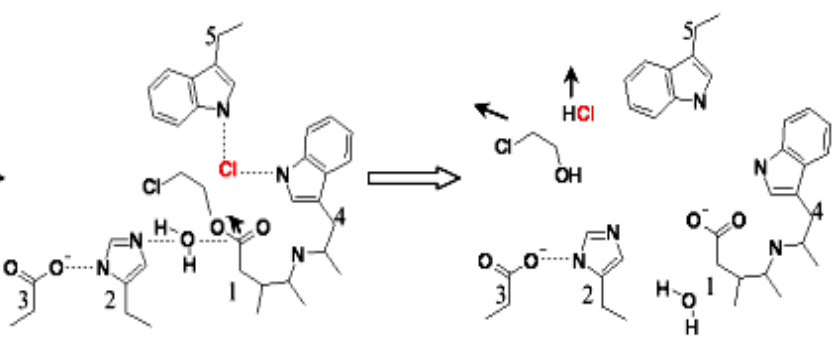

Hydrolysis
Product released

Fig. 2. Catalytic mechanism of HLDs. 1. nucleophilic aspartic acid, 2. histidine base, 3. glutamic/aspartic acid, 4. first halide stabilizing residue adjacent to nucleophilic, and 5 . second halide stabilizing residue. 


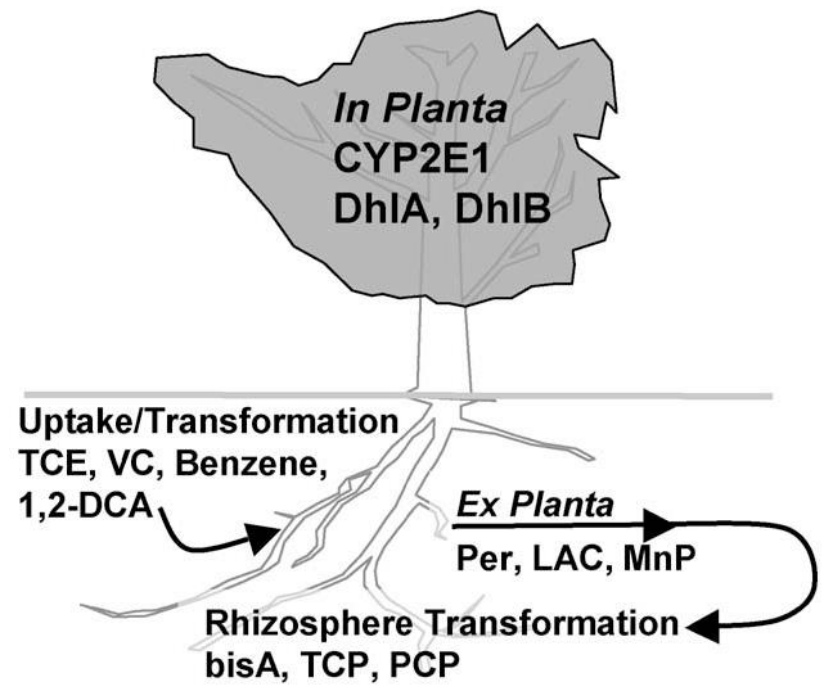

Fig. 3. The phytoremediation was conducted by introducing a hld and had genes into plants. The transgenic plant was able to absorb and detoxify some toxic halogenated compounds [37]

\section{Industrial application}

Due to the abundance and toxicity of halogenated compounds, it is not surprising that HLDs play a crucial potential role in processing aids in many biotechnological applications. Practical applications of HLDs can be performed in two approaches: (1) microbial dehalogenation, microorganism which is in nature or recombinant, contains HLDs gene, and (2) enzymatic dehalogenation, a directly used of isolated HLDs. In general, the most common application of HLDs is in biocatalysis, biotransformation, biodegradation, environmental bioremediation, biosensor and molecular imaging [21,32].

Even though alcohol can be produced naturally by microorganism or traditional organic synthesis, application of HLD provides several benefits that cannot be obtained by nature or organic synthesis. Probing the usefulness of HLDs for biocatalysis and biotransformation have been focused on synthesizes a chemical intermediate or end-product, especially alcohol with both dehalogenation approaches. For instance, efficiency and high enantioselectivity of HLDs, such as DhlA, LinB, DbjA [33] or DatA [7], have potential application to the synthesis of chiral compounds needed by pharmaceutical industry.

The potential for bioremediation of recalcitrant and hazardous halogenated pollutants from environment [31-32] and decontamination of chemical weapons [33] by HLDs has been proposed. The aim of decontamination is to rapidly and effectively render harmless or remove poisonous substances. Even though, both dehalogenation approaches can be implemented for cleaning-up environmental, microbial dehalogenation is preferred for environmental bioremediation because HLD isolation step can be skipped and this approach can be used in situ. In contrast, decontamination of chemical weapons must be performed at strictly isolated tank, thus, enzymatic dehalogenation to detoxify these toxic compounds is strictly recommended.

The potential application of HLD for phytoremediation [34] or as selectable marker [35] has been reported. A synergy of integrated two dehalogenases (HLD and haloacid dehalogenase, HAD) and endogenous dehydrogenases resulted a dehalogenase-transgenic plant is able to metabolize 1,2-dichloroethane to 2-chloroacetic acid. This result represents a significant advance in the development of a lowcost bioremediation technique and selectable marker toward the clean-up of halogenated organic pollutants from contaminated soil and groundwater (Fig. 3).

The applications of HLD for biosensor [36] and molecular imaging [37] have been developed. HLD based-biosensor mostly developed by microbial cells containing HLD gene because easy, cheap and no isolation and purification step during fabrication are required. However, this type of biosensor has low sensitivity, selectivity, stability. Second type of biosensor is developed by incorporating of coimmobilization of HLDs and a fluorescence $\mathrm{pH}$ indicator on the tip of an optical fiber. This biosensor can compensate all probelms that exhibit by microbial cells biosensor [36]. Molecular imaging system is developed based on protein tagging system that linked onto a single genetic fusion, either in solution, living cells or chemically fixed cells. HLDs as molecular imaging designed to covalently bind in highly specific and irreversible manners to ligands that comprise halogenated-attached molecules. The HLD-based molecular imaging system can demonstrate a multiple processes of cellular physiology such as protein translocation, proteinprotein interaction and protein-DNA complex [37]

\section{References}

1. D. O'Hagan and J. W. Schmidberger, Enzymes that catalyse $S_{N} 2$ reaction mechanisms, Nat. Prod. Rep. 27 (2010) 900-918.

2. V. A. Streltsov, Z. Prokop, J. Damborsky, Y. Nagata, A. Oakley and M. C. J. Wilce, Haloalkane dehalogenase LinB from Sphingomonas paucimobilis UT26: X-ray crystallographic studies of dehalogenation of brominated substrates, Biochemistry 42 (2003) 10104-10112.

3. Z. Prokop, M. Monincova, R. Chaloupkova, M. Klvana, Y. Nagata, D. B. Janssen et al., Catalytic mechanism of the haloalkane dehalogenase LinB from Sphingomonas paucimobilis UT26, J. Biol. Chem. 278 (2003) 45094-45100.

4. S. Keuning, D. B. Janssen and B. Witholt, Purification and characterization of hydrolytic haloalkane dehalogenase from Xanthobacter autotrophicus GJ10, J. Bacteriol. 163 (1985) 635-639.

5. J. Newman, T. S. Peat, R. Richard, L. Kan, P. E. Swanson, J. A. Affholter, J. A. et al., Haloalkane dehalogenases: Structure of a Rhodococcus enzyme, Biochemistry 38 (1999) 16105-16114.

6. A. Jesenska, I. Sedlacek and J. Damborsky, Dehalogenation of Haloalkanes by Mycobacterium tuberculosis H37Rv and Other Mycobacteria, Appl. Environ. Microbiol. 66 (2000) 219-222.

7. K. Hasan, A. Fortova, T. Koudelakova, R. Chaloupkova, M. Ishitsuka, Y. Nagata, et al., 2011. Biochemical characteristics of the novel haloalkane dehalogenase DatA, isolated from the plant pathogen Agrobacterium tumefaciens C58, Appl. Environ. Microbiol. 77 (2011) 1881-1884.

8. I. Drienovska, E. Chovancova, T. Koudelakova, J. Damborsky and R. Chaloupkova, Biochemical characterization of a novel haloalkane dehalogenase from a cold-adapted bacterium, Appl. Environ. Microbiol. 78 (2012) 4995-4998.

9. S. M. Franken, H. J. Rozeboom, K. Kalk and B. W. Dijkstra, Crystal structure of haloalkane dehalogenase: an enzyme to detoxify halogenated alkanes, EMBO J. 10 (1991) 1297-1302.

10. P. A. Mazumdar, J. C. Hulecki, M. M. Cherney, C. R. Garen and M. N. 
G. James, X-ray crystal structure of Mycobacterium tuberculosis haloalkane dehalogenase Rv2579, Biochim. Biophys. Acta. 1784 (2008) 351-362.

11. Z. Prokop, Y. Sato, J. Brezovsky, T. Mozga, R. Chaloupkova, T. Koudelakova et al., Enantioselectivity of haloalkane dehalogenases and its modulation by surface loop engineering, Angew. Chem. Int. Ed. 49 (2010) 6111-61115.

12. M. Hesseler, X. Bogdanovic, A. Hidalgo, J. Berenguer, G. J. Palm, W. Hinrichs et al, Cloning, functional expression, biochemical characterization, and structural analysis of a haloalkane dehalogenase from Plesiocystis pacifica SIR-1, Appl. Microbiol. Biotechnol. 91 (2011) 1049-1060.

13. J. J. Gehret, L. Gu, T. W. Geders, W. C. Brown, L. Gerwick, W. H. Gerwick, W. H., et al., Structure and activity of DmmA, a marine haloalkane dehalogenase, Protein Sci. 21 (2012) 239-248.

14. L. Guan, H. Yabuki, M. Okai, J. Ohtsuka and M. Tanokura, Crystal structure of the novel haloalkane dehalogenase DatA from Agrobacterium tumefaciens $C 58$ reveals a special halide-stabilizing pair and enantioselectivity mechanism, Appl. Microbiol. Biotechnol. 98 (2014) 8573-8582.

15. J. Marek, J. Vevodova, I. Kuta-Smatanova, Y. Nagata, L. A. Svensson, J. Newman et al., Crystal structure of the haloalkane dehalogenase from Sphingomonas paucimobilis UT26, Biochemistry 39 (2000) 1408214086.

16. K. H. Verschueren, F. Seljee, H. J. Rozeboom, K. H. Kalk and B. W. Dijkstra, Crystallographic analysis of the catalytic mechanism of haloalkane dehalogenase, Nature 363 (1993) 693-698.

17. S. Barth, M. Fischer, R. D. Schmid and J. Pleiss, The database of epoxide hydrolases and haloalkane dehalogenases: one structure, many functions, Bioinformatics. 20 (2004) 2845-2847.

18. M. Nardini and B. W. Dijkstra, $\alpha / \beta$-hydrolase fold enzymes: the family keeps growing, Curr. Opin. Struct. Biol. 9 (1999) 732-737.

19. D. L. Ollis, E. Cheah, M. Cygler, B. Dijkstra, F. Frolow, S. M. Franken et al., 1992. The $\alpha / \beta$ hydrolase fold, Protein Eng. Des. Sel. 5 (1992) 197211

20. M. Holmquist, Alpha/beta-hydrolase fold enzymes: structures, functions and mechanisms, Curr. Protein Pept. Sci. 1 (2000) 209-235.

21. T. Koudelakova, E. Chovancova, J. Brezovsky, M. Monincova, A. Fortova, J. Jarkovsky et al., Substrate specificity of haloalkane dehalogenases, Biochem. J. 435 (2011) 345-354.

22. E. Chovancova, J. Kosinski, J. M. Bujnicki and J. Damborsky, Phylogenetic analysis of haloalkane dehalogenases, Proteins. 67 (2007) 305-316.

23. J. Kmunicek, K. Hynkova, T. Jedlicka, Y. Nagata, A. Negri, F. Gago, et al., Quantitative analysis of substrate specificity of haloalkane dehalogenase LinB from Sphingomonas paucimobilis UT26, Biochemistry. 44 (2005) 3390-3401.

24. M. Otyepka and J. Damborsky, Functionally relevant motions of haloalkane dehalogenases occur in the specificity-modulating cap domains, Protein Sci. 11 (2002) 1206-1217.

25. J. P. Schanstra and D. B. Janssen, Kinetics of halide release of haloalkane dehalogenase: Evidence for a slow conformational change, Biochemistry. 35 (1996) 5624-5632.

26. G. H. Krooshof, R. Floris, A. W. J. W. Tepper and D. B. Janssen, Thermodynamic analysis of halide binding to haloalkane dehalogenase suggests the occurrence of large conformational changes, Protein Sci. 8 (1999) 355-360.

27. M. Bohac, Y. Nagata, Z. Prokop, M. Prokop, M. Monincova, M. Tsuda et al., Halide-stabilizing residues of haloalkane dehalogenases studied by quantum mechanic calculations and site-directed mutagenesis, Biochemistry. 41 (2002) 14272-14280.

28. J. Damborsky, R. Chaloupkova, M. Pavlova, E. Chovancova and J. Brezovsky, Structure-Function Relationships and Engineering of Haloalkane Dehalogenases. In: Handbook of Hydrocarbon and Lipid Microbiology, Kenneth N. Timmis (Ed.), Springer-Verlag, Berlin, Heidelberg, 2009, pp. 1081-1098.

29. F. Pries, J. Kingma, G. H. Krooshof, C. M. Jeronimus-Stratingh, A. P. Bruins and D. B. Janssen, Histidine 289 is essential for hydrolysis of the alkyl-enzyme intermediate of haloalkane dehalogenase, J. Biol. Chem. 270 (1995) 10405-10411.

30. K. Hynkova, Y. Nagata, M. Takagi and J. Damborsky, Identification of the catalytic triad in the haloalkane dehalogenase from Sphingomonas paucimobilis UT26, FEBS Lett. 446 (1999) 177-181.

31. D. B. Janssen, J. E. Oppentocht and G. J. Poelarends, Microbial dehalogenation, Curr. Opin. Biotechnol. 12 (2001) 254-258.

32. S. Fetzner and F. Lingens, Bacterial dehalogenases: biochemistry, genetics, and biotechnological applications, Microbiol. Rev. 58 (1994) 641-685.

33. Z. Prokop, F. Oplustil, J. DeFrank and J. Damborsky, Enzymes fight chemical weapons, Biotechnol. J. 1 (2006) 1370-1380

34. G. L. Mena-Benitez, F. Gandia-Herrero, S. Graham, T. R. Larson, S. J. McQueen-Mason, C. E. French et al., Engineering a catabolic pathway in plants for the degradation of 1,2-dichloroethane, Plant Physiol. 147 (2008) 1192-1198.

35. H. Naested, M. Fennema, L. Hao, M. Andersen, D. B. Janssen and J. Mundy, A bacterial haloalkane dehalogenase gene as a negative selectable marker in Arabidopsis, Plant J. 18 (1999) 571-576.

36. S. Bidmanova, R. Chaloupkova, J. Damborsky and Z. Prokop, Development of an enzymatic fiber-optic biosensor for detection of halogenated hydrocarbons, Anal. Bioanal. Chem. 398 (2010) 1891-1898.

37. G. V. Los, L. P. Encell, M. G. McDougall, D. D. Hartzell, N. Karassina, C. Zimprich et al., HaloTag: a novel protein labeling technology for cell imaging and protein analysis, ACS Chem. Biol. 3 (2008) 373-382.

38. C. A. James and S. E. Strand, Phytoremediation of small organic contaminants using transgenic plants. Current opinion in biotechnology, 20 (2009) 237-41. 